\title{
Period spacings in red giants
}

\section{Disentangling rotation and revealing core structure discontinuities}

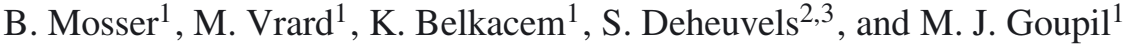 \\ 1 LESIA, Observatoire de Paris, PSL Research University, CNRS, Université Pierre et Marie Curie, Université Paris Diderot, \\ 92195 Meudon, France \\ e-mail: benoit.mosser@obspm.fr \\ 2 Université de Toulouse, UPS-OMP, IRAP, 31400 Toulouse, France \\ ${ }^{3}$ CNRS, IRAP, 14 avenue Edouard Belin, 31400 Toulouse, France
}

Received 28 July 2015 / Accepted 16 September 2015

\begin{abstract}
Context. Asteroseismology allows us to probe the physical conditions inside the core of red giant stars. This process relies on the properties of the global oscillations with a mixed character that are highly sensitive to the physical properties of the core. However, overlapping rotational splittings and mixed-mode spacings result in complex structures in the mixed-mode pattern, which severely complicates its identification and the measurement of the asymptotic period spacing.

Aims. This work aims at disentangling the rotational splittings from the mixed-mode spacings in order to open the way to a fully automated analysis of large data sets.

Methods. An analytical development of the mixed-mode asymptotic expansion is used to derive the period spacing between two consecutive mixed modes. The échelle diagrams constructed with the appropriately stretched periods are used to exhibit the structure of the gravity modes and of the rotational splittings.

Results. We propose a new view of the mixed-mode oscillation pattern based on corrected periods, called stretched periods, that mimic the evenly spaced gravity-mode pattern. This provides a direct understanding of all oscillation components, even in the case of rapid rotation. In this way, the measurement of the asymptotic period spacing and the signature of the structural glitches on mixed modes can be performed easily.

Conclusions. This work makes it possible to derive all seismic global parameters in an automated way, including the identification of the different rotational multiplets and the measurement of the rotational splitting, even when this splitting is significantly larger than the period spacing. Revealing buoyancy glitches provides a detailed view of the radiative core.
\end{abstract}

Key words. stars: oscillations - stars: interiors - stars: evolution

\section{Introduction}

Asteroseismic observations obtained with CoRoT and Kepler have delivered a great deal of information on the stellar interior structure (e.g., Michel et al. 2008; Chaplin et al. 2011), especially for red giants (e.g., De Ridder et al. 2009; Bedding et al. 2010). Independent of modeling, global seismic properties provide relevant estimates of the stellar masses and radii (e.g., Kallinger et al. 2010; Mosser et al. 2010). The dipole modes with a mixed character probe the core and test the evolutionary status of the stars (Bedding et al. 2011; Mosser et al. 2011a). They also provide the measurement of the asymptotic period spacing (Mosser et al. 2012b) which is directly related to the core mass (Montalbán et al. 2013). Their observation also gives access to the differential-rotation profile in red giants (Beck et al. 2012). The measurement of the mean core rotation for about 300 stars analyzed by Mosser et al. (2012c), and extended toward a few subgiants (Deheuvels et al. 2014), indicates that angular momentum must be efficiently transferred from the stellar core to the envelope (e.g., Marques et al. 2013). At this stage, explaining the spinning-down remains difficult, but a recent work by Belkacem et al. (2015b,a) shows that mixed modes likely participate in the angular-momentum transfer and induce the slowing down of the core rotation.
All these results illustrate the ability of the mixed-mode oscillations to probe the innermost radiative core and deliver unique information on the physical conditions deep inside the star. This emphasizes the need for new observational constraints on data sets that are as large as possible. As more than 13000 red giant oscillation spectra observed by Kepler show solar-like oscillations (Stello et al. 2013), rotation could be measured in a much larger data set than previously done (Mosser et al. 2012c). This would enable us to test in detail how the mean core rotation and the angular momentum transfer vary with stellar evolution.

To date, precise rotational splittings have mostly been manually determined. Their automated measurement is possible only when the rotational frequency is much smaller than the mixedmode spacing. This condition is most often met for clump stars, but rarely on the red giant branch (RGB). We seek a rapid, effective, and automated method for measuring this parameter in thousands of stars. This first requires a way to disentangle rotational splittings and period spacings, then an automated measurement of the asymptotic period spacing. The method recently proposed by Datta et al. (2015) provides a partial answer for measuring the period spacing, applicable only to non-rotating stars or to stars observed pole-on.

As is well known, rapid interior structure variations affect the sound-speed and Brunt-Väisälä frequency profiles, so that they 
modify the regular arrangement of both pure pressure and pure gravity modes, hence of the mixed-mode pattern. Such soundspeed glitches are clearly identified in red giant oscillations (Miglio et al. 2010; Corsaro et al. 2015; Vrard et al. 2015a); they arise from the second helium ionization zone (e.g., Broomhall et al. 2014). Their effect on the radial oscillation modes remains limited to minor frequency changes compared to the large separation, but it is enough to induce a clear difference between stars on the RGB or in the red clump. This difference was observationally shown by Kallinger et al. (2012) and theoretically studied by Christensen-Dalsgaard et al. (2014). A systematic survey by Vrard et al. (2015a) firmly assesses this difference and shows that it is created by acoustic glitches in the external envelope related to the second ionization of helium.

Buoyancy glitches are also expected in red giants as a consequence of an interior structure gradient due to a local phenomenon related to nuclear burning or mixing (Cunha et al. 2015). To date, they have not been observed. However, as suggested by Cunha et al. (2015), they may explain cases where the identification of the mixed-mode pattern is difficult or not possible.

In this work, with a new expression of the second-order asymptotic expansion for dipole mixed modes, we analyze the structure of their oscillation pattern including rotation, soundspeed glitches, or buoyancy glitches. In Sect. 2 we show that the period spacing and the rotational splitting are constructed on similar patterns: both are bumped near the pressure dominated modes. The rotational pattern was formerly derived by Deheuvels et al. (2015). Consequences of this new result are discussed in Sect. 3 where we show how the structure of the pure gravity-mode pattern can be revealed from the mixed-mode pattern. Section 4 shows how rotation can be fully disentangled from the mixed mode pattern, even in the most complex cases where rotational splittings and period spacings largely overlap (see, e.g., Figs. A.4-A.6 of Mosser et al. 2012c). We then investigate the principle of the full automation of the analysis of the red giant oscillation spectrum and test the influence of structure glitches in Sect. 5.

A companion paper, Vrard et al. (2015b), presents the automated method for deriving the asymptotic period spacing in Kepler red giants, which is based on the present work.

\section{Mixed mode period spacing}

Following the work of Shibahashi (1979) and Unno et al. (1989), asymptotic expansions of mixed modes have been derived for different seismic parameters: eigenfrequencies (Mosser et al. 2012b), period spacings (Christensen-Dalsgaard 2012), and rotational splittings (Goupil et al. 2013; Deheuvels et al. 2015).

Here, we intend to provide expansions taking into account complex expressions of the pure pressure and pure gravity contributions.

\subsection{Asymptotic expansion}

Shibahashi (1979) and Unno et al. (1989) derived an implicit asymptotic relation for mixed modes, which expresses as

$\tan \theta_{\mathrm{p}}=q \tan \theta_{\mathrm{g}}$,

where $q$ is the coupling factor between the gravity and pressure components of the modes and where the phase $\theta_{\mathrm{p}}$ and $\theta_{\mathrm{g}}$ refer, respectively, to the pressure- and gravity-wave contributions. At first order, the phases for dipole modes are related to the period spacing $\Delta \Pi_{1}$ and large separation $\Delta v$, respectively. They write

$\theta_{\mathrm{g}}=\pi\left(\frac{1}{v \Delta \Pi_{1}}-\varepsilon_{\mathrm{g}}\right)$,
$\theta_{\mathrm{p}}=\pi\left(\frac{v}{\Delta v}-\frac{1}{2}-\varepsilon_{\mathrm{p}}\right)$,

as used for red giants by Mosser et al. (2012b), but here correctly accounting for the contribution $\ell / 2$ with $\ell=1$, and for the offsets $\varepsilon_{\mathrm{g}}$ and $\varepsilon_{\mathrm{p}}$.

Owing to the form of the mixed-mode asymptotic expansion, we can modify the phases in Eqs. (2) and (3) with additional terms, provided their contributions are multiples of $\pi$. This is a useful artifice for introducing the eigenfrequencies of either pure pressure or pure gravity modes. Therefore, we rewrite the phases as

$$
\begin{aligned}
& \theta_{\mathrm{g}}=\pi \frac{1}{\Delta \Pi_{1}}\left(\frac{1}{v}-\frac{1}{v_{\mathrm{g}}}\right), \\
& \theta_{\mathrm{p}}=\pi \frac{v-v_{\mathrm{p}}}{\Delta v\left(n_{\mathrm{p}}\right)},
\end{aligned}
$$

where $v_{\mathrm{p}}$ and $v_{\mathrm{g}}$ are the asymptotic frequencies of pure pressure and gravity modes, respectively, and $\Delta v\left(n_{\mathrm{p}}\right)$ is the frequency difference between the consecutive pure pressure radial modes with radial orders $n_{\mathrm{p}}$ and $n_{\mathrm{p}}+1$. The offsets and $\ell / 2$ term introduced in Eqs. (2) and (3) are included in the terms $v_{\mathrm{p}}$ and $v_{\mathrm{g}}$. This means that we now have the possibility of using Eqs. (4) and (5) at any order of the asymptotic expansions for the pure pressure and gravity contributions.

For $v_{\mathrm{g}}$, acceptable fits of red giant oscillation spectra are based on the first-order asymptotic expansion (Tassoul 1980). For dipole modes, we have

$\frac{1}{v_{\mathrm{g}}}=\left(-n_{\mathrm{g}}+\varepsilon_{\mathrm{g}}\right) \Delta \Pi_{1}$

where $n_{\mathrm{g}}$ is the gravity radial order, usually defined with a negative value, and the offset $\varepsilon_{\mathrm{g}}=1 / 4-\varepsilon_{\mathrm{g}}{ }^{\prime}$. The gravity offset $\varepsilon_{\mathrm{g}}{ }^{\prime}$ is a small but complicated function sensitive to the stratification near the boundary between the radiative core and the convective envelope (Provost \& Berthomieu 1986). If needed, in Eq. (4) we can consider a second-order expansion for the gravity expansion (Tassoul 1980) or the contribution of buoyancy glitches (e.g., Miglio et al. 2008; Cunha et al. 2015). This is done in Sect. 5.

For $v_{\mathrm{p}}$, the high quality of the seismic data requires the use of the second-order asymptotic expansion (Mosser et al. 2013). For dipole modes, we have

$v_{\mathrm{p}}\left(n_{\mathrm{p}}\right)=\left(n_{\mathrm{p}}+\frac{1}{2}+\varepsilon_{\mathrm{p}}+\frac{\alpha}{2}\left(n_{\mathrm{p}}-n_{\max }\right)^{2}+d_{01}\right) \Delta v$,

where $n_{\mathrm{p}}$ is the pressure radial order, $\alpha$ represents the curvature of the radial oscillation pattern, $n_{\max }=v_{\max } / \Delta v-\varepsilon_{\mathrm{p}}$ is the non-integer order at the frequency $v_{\max }$ of maximum oscillation signal, and $d_{01}$ is the small separation, namely the distance, in units of $\Delta v$, of the pure pressure dipole mode compared to the midpoint between the surrounding radial modes (Mosser et al. 2013). In this case, the observed large separation $\Delta v\left(n_{\mathrm{p}}\right)$ in Eq. (5) increases with increasing radial order,

$\Delta v\left(n_{\mathrm{p}}\right)=\Delta v\left(1+\alpha\left(n_{\mathrm{p}}-n_{\max }\right)\right)$,

which is obtained from the derivation of Eq. (7). For the gravity contribution, the asymptotic expressions of the pure $p$ modes 
can be as precise as required; $v_{\mathrm{p}}$ may include the glitch component due to the second ionization of helium (Miglio et al. 2010; Broomhall et al. 2014; Vrard et al. 2015a).

\subsection{Bumped period spacing}

In order to retrieve the relative period spacing $\Delta P / \Delta \Pi_{1}$ between two consecutive mixed modes, the mixed-mode radial order, $n_{\mathrm{m}}=n_{\mathrm{p}}+n_{\mathrm{g}}$, needs to be introduced. We then choose to write the period $P=1 / v$ of a mixed mode as

$P=n_{\mathrm{m}} \Delta \Pi_{1}+p$,

where the residual term $p$ expresses the departure to an evenly spaced comb function. This period is introduced in the phases $\theta_{\mathrm{g}}$ and $\theta_{\mathrm{p}}$ provided by Eqs. (4) and (5) and linked by the asymptotic relation (Eq. (1)). In the gravity contribution, all terms multiple of $\pi$ cancel out owing to the property of the tangent function, so that with Eq. (9) the term $\tan \theta_{\mathrm{g}}$ reduces to $\tan \pi\left(p / \Delta \Pi_{1}-\varepsilon_{\mathrm{g}}\right)$. The derivation of Eq. (1) with respect to $n_{\mathrm{m}}$ then gives

$\frac{\frac{\mathrm{d}}{\mathrm{d} n_{\mathrm{m}}}\left(\frac{1}{n_{\mathrm{m}} \Delta \Pi_{1}+p}\right)}{\Delta v\left(n_{\mathrm{p}}\right) \cos ^{2} \theta_{\mathrm{p}}}=q \frac{\frac{\mathrm{d} p}{\mathrm{~d} n_{\mathrm{m}}}}{\Delta \Pi_{1} \cos ^{2} \theta_{\mathrm{g}}}$

when the variation of $\Delta v\left(n_{\mathrm{p}}\right)$ with frequency is neglected in the pressure term. One easily obtains

$-v^{2} \frac{\mathrm{d} P}{\mathrm{~d} n_{\mathrm{m}}}=q \frac{\Delta v\left(n_{\mathrm{p}}\right)}{\Delta \Pi_{1}} \frac{\cos ^{2} \theta_{\mathrm{p}}}{\cos ^{2} \theta_{\mathrm{g}}}\left[\frac{\mathrm{d} P}{\mathrm{~d} n_{\mathrm{m}}}-\Delta \Pi_{1}\right]$,

hence

$\frac{1}{\Delta \Pi_{1}} \frac{\mathrm{d} P}{\mathrm{~d} n_{\mathrm{m}}}=\left[1+\frac{1}{q} \frac{v^{2} \Delta \Pi_{1}}{\Delta v\left(n_{\mathrm{p}}\right)} \frac{\cos ^{2} \theta_{\mathrm{g}}}{\cos ^{2} \theta_{\mathrm{p}}}\right]^{-1}$.

The case $\mathrm{d} n_{\mathrm{m}}=1$ corresponds to the period difference between two consecutive mixed modes, and thus provides us with the relative bumped period spacing

$\frac{\Delta P}{\Delta \Pi_{1}}=\zeta(v)$

where the definition

$\zeta(v)=\left[1+\frac{1}{q} \frac{v^{2} \Delta \Pi_{1}}{\Delta v\left(n_{\mathrm{p}}\right)} \frac{\cos ^{2} \pi \frac{1}{\Delta \Pi_{1}}\left(\frac{1}{v}-\frac{1}{v_{\mathrm{g}}}\right)}{\cos ^{2} \pi \frac{v-v_{\mathrm{p}}}{\Delta v\left(n_{\mathrm{p}}\right)}}\right]^{-1}$

matches the first-order asymptotic expansion derived by Deheuvels et al. (2015) for expressing the mixed-mode rotational splitting

$\delta v_{\mathrm{rot}}=\delta v_{\mathrm{rot}, \mathrm{g}} \zeta+\delta v_{\mathrm{rot}, \mathrm{p}}(1-\zeta)$

where $\delta v_{\text {rot,g }}$ and $\delta v_{\text {rot,p }}$ are the rotational splittings related to pure gravity or pure pressure modes. In the limit case where the mean envelope rotation is negligible compared to the mean core rotation, this simplifies into

$\zeta(v) \simeq \frac{\delta v_{\mathrm{rot}}}{\delta v_{\mathrm{rot}, \mathrm{g}}}$

Hence, we have demonstrated that the period spacing compared to $\Delta \Pi_{1}$ (Eq. (13)) and the rotational splitting compared to $\delta v_{\text {rot, }}$ (Eq. (16)) follow the same distribution. This result emphasizes

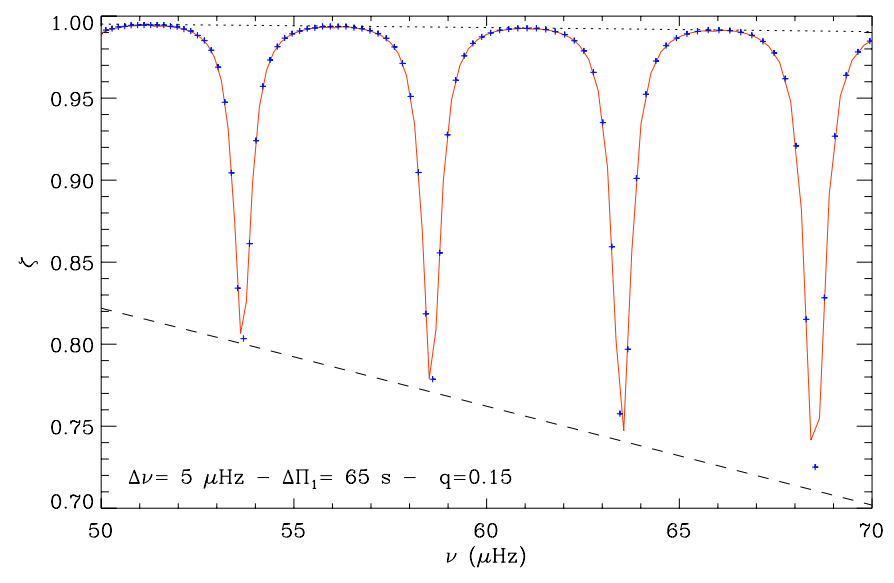

Fig. 1. Relative period spacings $\Delta P / \Delta \Pi_{1}$ and relative rotational splittings $\zeta$ for a synthetic RGB mixed-mode spectrum. The red line shows the asymptotic $\zeta$ function; the blue crosses represent the period spacings derived from the asymptotic expansion of mixed modes and scaled to $\Delta \Pi_{1}$. The dashed line shows the minimum values $\zeta_{\min }$ reached by pressure-dominated mixed modes; the dotted line shows the maximum values $\zeta_{\max }$ reached by gravity-dominated mixed modes.

that the coupling between the pressure and gravity terms plays the same role for arranging the mixed-mode periods and the frequency rotational splittings. As shown in Goupil et al. (2013) and Deheuvels et al. (2015), this behavior is governed by the inertia of the mixed modes: $\zeta$ is the ratio between the kinetic energy in the radiative cavity and the total kinetic energy. The equality between $\Delta P / \Delta \Pi_{1}$ and $\zeta$ is seen in Fig. 1, where the bumped period spacings $\Delta P$ defined as $P\left(n_{\mathrm{m}}+1\right)-P\left(n_{\mathrm{m}}\right)$ are compared to the function $\zeta$. In order to avoid quantization biases, we have plotted $\Delta P / \Delta \Pi_{1}$ at the abscissae $\left(P\left(n_{\mathrm{m}}\right)+P\left(n_{\mathrm{m}}+1\right)\right) / 2$.

We must note that the values of $\zeta$ obtained for the discrete mixed-mode frequencies $v$ do not reflect the complexity of the function $\zeta$ if considered as a continuous function of frequency. For clarity, we denote $\zeta(v)$ the discrete values and $\zeta(f)$ the continuous function. The contributions of the phases $\theta_{\mathrm{p}}$ and $\theta_{\mathrm{g}}$ to $\zeta(f)$ are shown in Fig. 2.

Interestingly, the pattern $\zeta(v)$ reached for mixed modes mainly depends on the asymptotic parameters of the pure pressure modes, since the location of the local minima only depends on the frequency of the pure pressure dipole modes. Conversely, it hardly depends on $\Delta \Pi_{1}$ and $q$ (Vrard et al. 2015b). Limiting cases corresponding to gravity-dominated mixed modes ( $g$ - $m$ mode) and pressure-dominated mixed modes ( $p$ - $m$ mode) are derived in the Appendix.

\subsection{Comparison with previous work}

Recently, Benomar et al. (2014) remarked that the ratio $I_{1} / I_{0}$ of the inertia of dipole modes compared to radial modes may provide an alternative to using mixed-mode frequencies and give essentially the same information as the mixed-mode asymptotic equation. We confirm that this is the case: their function $I_{1} / I_{0}$ can be related to $\zeta$ if we assume that the difference between the total inertia of mixed modes and the contribution of the radiative cavity only exactly corresponds to the inertia of radial modes. Their Eq. (10), with the factor $q^{2} / 4$ corrected to $q$, can be written

$\frac{I_{1}}{I_{0}}=\frac{1}{1-\zeta}$ 


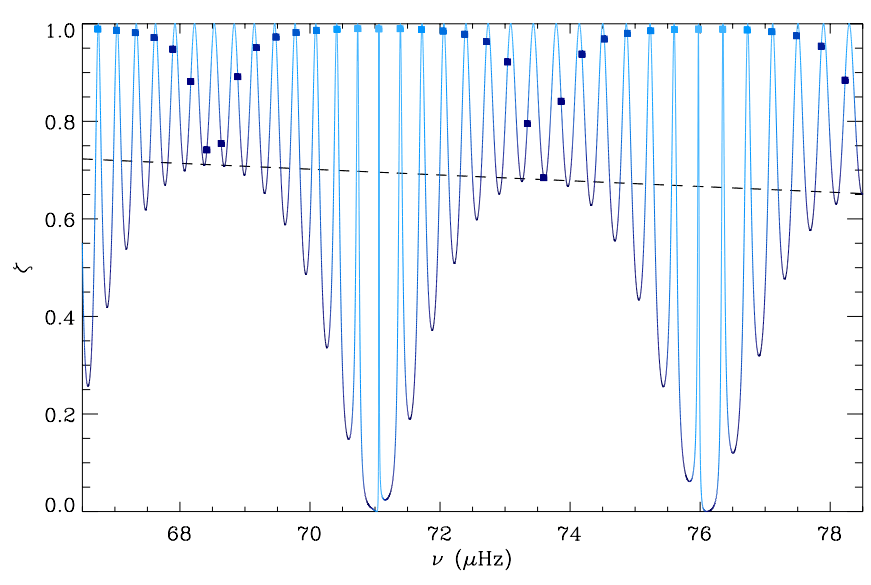

Fig. 2. Comparison of the function $\zeta$ considered as a continuous function of frequency (continuous line with color modulated by the value of $\cos ^{2} \theta_{\mathrm{g}}$ ) with the values derived for the mixed-mode frequencies (full squares with color modulated by the value of $\cos ^{2} \theta_{\mathrm{p}}$ ). Values of $\cos ^{2}$ close to 0 and 1 appear in light blue and dark blue, respectively. For gravity-dominated mixed modes, the phase term $\theta_{\mathrm{p}}$ acts for $\zeta$ values close to the local maxima; for pressure-dominated mixed modes, the $\zeta$ values are close to the local minima $\zeta_{\min }$ (dashed line). The simulation is based on the second-order asymptotic pressure-mode pattern proposed for red giants by Mosser et al. (2013), with $\Delta v=5 \mu \mathrm{Hz}$, and on the first-order asymptotic expansion of gravity modes, with $\Delta \Pi_{1}=65 \mathrm{~s}$ and $\varepsilon_{\mathrm{g}}=0$.

A different expression of the bumped period spacing was earlier introduced by Christensen-Dalsgaard (2012). Rewriting his Eq. (34) with our notation gives

$\frac{\Delta P}{\Delta \Pi_{1}}=\left[1-\frac{v^{2} \Delta \Pi_{1}}{\pi} \frac{\mathrm{d} \Phi}{\mathrm{d} v}\right]^{-1}$,

with $\Phi$ defined in Christensen-Dalsgaard (2012) by

$\tan \Phi=\frac{q}{\tan \theta_{\mathrm{p}}}$

By comparison with Eq. (1), we get $\Phi \equiv \pi / 2-\theta_{\mathrm{g}}$. The term $\mathrm{d} \Phi / \mathrm{d} v$ derived from Eq. (19) ensures the agreement between the different expressions of $\Delta P / \Delta \Pi_{1}$. We note that the use of Eq. (14) avoids the introduction of the derivative term present in Eq. (18). As rapid variations arise from the $\theta_{\mathrm{g}}$ contribution, our new expression of $\Delta P / \Delta \Pi_{1}$ (Eqs. (13) and (14)) provides a more precise numerical result than Eq. (18), which requires a numerical derivative.

\section{Stretched periods}

In this section, we show how the function $\zeta$ can be derived from the analysis of the pressure mode pattern and how it can be used to extract information on the pure gravity mode pattern.

\subsection{Identification of the mixed modes}

In a red giant oscillation spectrum, the location of the mixed modes is fixed by the measurement of the large separation $\Delta v$. The determination of $\Delta v$, first derived from the envelope autocorrelation function (Mosser \& Appourchaux 2009) and then refined with the universal red giant oscillation pattern (Mosser et al. 2011b), provides the identification of the radial modes and helps to locate the frequency ranges where mixed modes cannot

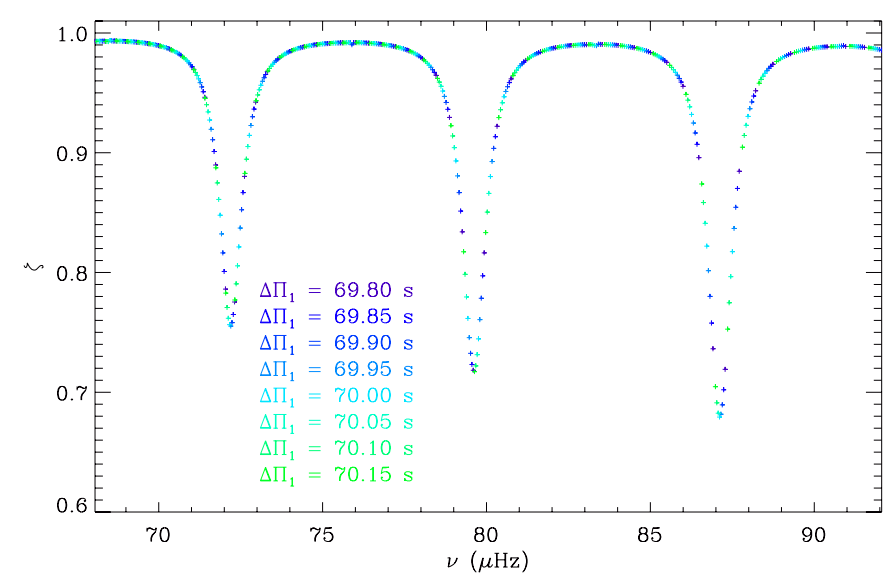

Fig. 3. Interpolation of the function $\zeta$ for the period spacing $\Delta \Pi_{1}$ derived from the values obtained with various periods in the range $\Delta \Pi_{1}(1 \pm$ $\left.v_{\max } \Delta \Pi_{1} / 2\right)$, where $v_{\max }$ is the frequency of maximum oscillation signal.

be mistaken for radial or quadrupole modes. The background parameters, derived as in Mosser et al. (2012a), are used to correct the granulation contribution in the frequency range around $v_{\max }$ where oscillations are observed. Hence, mixed modes can be automatically identified in frequency ranges that have no radial and quadrupole modes with a height significantly above the background.

\subsection{Estimate of the function $\zeta$}

The same information is used to derive the function $\zeta$. The precise location of the minima of the function is obtained from the location of the $p-m$ modes, which is fixed by the radial pattern. Then, basic information from the $\Delta \Pi_{1}-\Delta v$ diagram (Mosser et al. 2014) is enough to obtain a precise estimate of $\zeta$. Large modifications of $\Delta \Pi_{1}$ only slightly modify the $\zeta$ profile (Vrard et al. 2015b). Furthermore, the variation of $q$ with $\Delta \Pi_{1}$, hence with $\Delta v$, also obeys a deterministic relation, so that $q$ can be predicted with a precision better than $20 \%$, which is enough.

As shown in Fig. 2, the function $\zeta$ is properly defined only for the mixed-mode frequencies. Therefore, to obtain a continuous function representative of the period change due to the mixing of the modes, we have to interpolate the values of $\zeta(v)$. Figure 3 graphically explains how this can be obtained with a small modulation of the period spacing, assuming that $\zeta$ only slightly depends on $\Delta \Pi_{1}$. This property is fully developed in Vrard et al. (2015b).

\subsection{Stretched periods}

The interpolated form of $\zeta$ is then used to turn the frequencies into periods $\tau$ with the differential equation

$\mathrm{d} \tau=\frac{1}{\zeta} \frac{\mathrm{d} v}{v^{2}}$

from which we can integrate the periods $\tau$ of mixed modes (Fig. 4). The constant of integration can be arbitrarily fixed since it plays no role. Owing to the $\zeta$ profile (Fig. 1), we call them stretched periods. They are used for drawing échelle diagrams, where $v$ is plotted as a function of $\tau$ modulo $\Delta \Pi_{1}$. 
B. Mosser et al.: Period spacings in red giants. I.

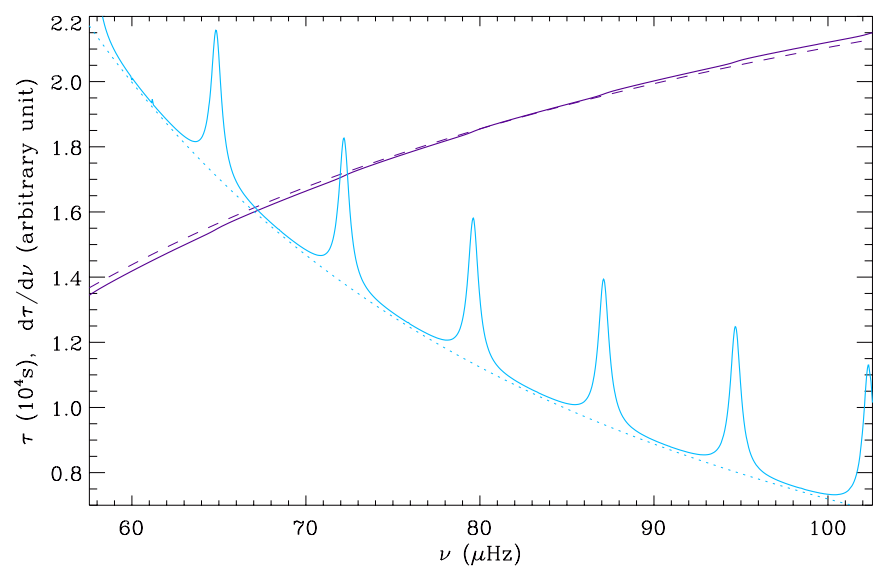

Fig. 4. Stretched period $\tau$ as a function of frequency (dark blue line) compared to a reference varying as $-1 / v$ (dashed line). The derivative $\mathrm{d} \tau / \mathrm{d} v$ is superimposed (light blue line) with an arbitrary scaling factor to fit in the same window as $\tau$; the dotted line represents the term $1 / v^{2}$.

\section{4. Échelle diagrams based on the stretched periods}

We first consider the case of a star seen pole-on, for which rotation is not an issue; the full case including rotation is solved in Sect. 4. The left panel of Fig. 5 shows the varying period spacings of the mixed modes. The échelle diagram based on the periods $1 / v$ (middle panel) shows the classical S-shape (Bedding et al. 2011; Mosser et al. 2012b), whereas the échelle spectrum based on the stretched periods $\tau$ (right panel) exhibits a single nearly vertical ridge where discrepant peaks can be easily identified as speckle structures due to the short-lived $p-m$ modes or as $\ell=3$ modes. As for classical échelle diagrams used to estimate the large separation $\Delta v$, the value of the period used to fold the stretched period influences the slope of the ridge. It is easy to ensure a vertical alignment and then extract the measurement of the period spacing $\Delta \Pi_{1}$. This is done in the companion paper (Vrard et al. 2015b) and we leave the discussion of the performance for measuring $\Delta \Pi_{1}$ to this work.

\subsection{Precise measurement of the coupling factor}

The precision of the measurement of the coupling term $q$ can be significantly enhanced. Previous estimates showed uncertainties as large as 0.05 for a mean value of $q$ about 0.2 (Mosser et al. $2012 \mathrm{~b}$ ). Here, the expression of $\zeta_{\text {min }}$ gives a new way to measure $q$ in an automated manner. As in Fig. 1, the period spacings for a fixed $m$ ridge can be compared with the function $\zeta_{\min } \Delta \Pi_{1}$, and $q$ can be derived with a smaller uncertainty. It is even possible to reduce this uncertainty to about 0.02 by tuning $q$ to ensure a minimum spread around $p-m$ modes in the échelle diagram constructed with the stretched periods.

\section{Disentangling rotation}

In this section we use the analytical expression of the period spacing (Eq. (14)) to disentangle the complex structure of the mixed mode pattern, especially for red giants on the RGB where the most complex cases are found (Mosser et al. 2012c). For this purpose, we have considered stars analyzed in Beck et al. (2012) and Mosser et al. (2012a,b,c). We have retrieved the public data set in the Kepler archive in order to benefit from the longest available time series (about 4 years).

\subsection{Rotational splittings}

The rotation is considered as a perturbation of the dipole mixedmode pattern and because the surface rotation is negligible in most cases, the unperturbed frequency $v$ is changed by rotation into $v-m \delta v_{\text {rot }}$ (Goupil et al. 2013). According to Eq. (15) and to the profile of $\zeta$, this reduces to $v-m \zeta \delta v_{\text {rot,core }}$, even in the cases where the envelope rotation cannot be neglected, for instance in the secondary clump (Deheuvels et al. 2015). For dipole modes, azimuthal orders $m \in\{-1,0,1\}$. Hence, the differences in the stretched periods of dipole modes with same azimuthal order $m$ can be approximated by

$\Delta \tau_{m}=\Delta \Pi_{1}\left(1+2 m \frac{\zeta \delta v_{\mathrm{rot}, \mathrm{core}}}{v}\right)$

if we assume that the mean envelope rotation can be omitted (Goupil et al. 2013). Even for an important rotational splitting as large as or even larger than the period spacing, the relative correction due to rotation is small. Therefore, the stretched period spacings $\Delta \tau_{m= \pm 1}$ are close to $\Delta \Pi_{1}$ (Table 1 ). Since modes are observed in a limited frequency range around the frequency $v_{\max }$ of maximum oscillation signal, we can approximate the varying term $\zeta / v$ of Eq. $(21)$ by $\mathcal{N} /\left((\mathcal{N}+1) v_{\max }\right)$, where the function

$\mathcal{N}(v)=\frac{\Delta v}{\Delta \Pi_{1} v^{2}}$

shows how the period spacings compare to the large separation $\Delta v$. It corresponds to the number of gravity modes per $\Delta v$ wide frequency range around the frequency $v$. This shows that the period spacing of each component of the rotation multiplet is nearly uniform, close to

$\Delta \tau_{m} \simeq \Delta \Pi_{1}\left(1+m x_{\text {rot }}\right)$,

with

$x_{\text {rot }}=2 \frac{\mathcal{N}\left(v_{\max }\right)}{\mathcal{N}\left(v_{\max }\right)+1} \frac{\delta v_{\text {rot,core }}}{v_{\text {max }}}$.

We note that the ratio $\mathcal{N} /(\mathcal{N}+1)$ is a consequence of the addition of an extra pressure mode among the $\mathcal{N}$ gravity modes that construct the $(\mathcal{N}+1)$ mixed modes in a $\Delta v$-wide frequency range.

\subsection{Measurement of $\Delta \Pi_{1}$ and $\delta v_{\text {rot,core }}$}

Equation (21) allows us to correct the variation of the rotational splitting with frequency and their asymmetry near the $p$ - $m$ modes. According to Eq. (23), each azimuthal order forms a well-identified ridge in the échelle diagram. Each ridge approximately shows linear variation of the rotationally perturbed period spacings (Fig. 6) from which we can derive the period spacing $\Delta \Pi_{1}$ and the rotational splitting $\delta v_{\text {rot }}$. When the rotation splitting exceeds half the mixed-mode spacing, the $m= \pm 1$ ridges intersect (Fig. 6, right panel). Results obtained for these stars are given in Table 1 . Values agree with the rotational splittings measured in Mosser et al. (2012c), with a small difference due to the approximation used in this earlier paper, which is explained by the difference between the approximate form of the rotational splitting found by Goupil et al. (2013) and the more precise asymptotic rotational splitting (Deheuvels et al. 2015).

When the surface rotation cannot be omitted, as is the case on the low RG (Deheuvels et al. 2012, 2014) or in the secondary red clump (Deheuvels et al. 2015), the rotation correction $\zeta \delta v_{\text {rot }}$ in Eq. (21) must be changed into $\zeta\left(\delta v_{\text {rot,core }}-\delta v_{\text {rot,env }}\right)+\delta v_{\text {rot,env }}$ 

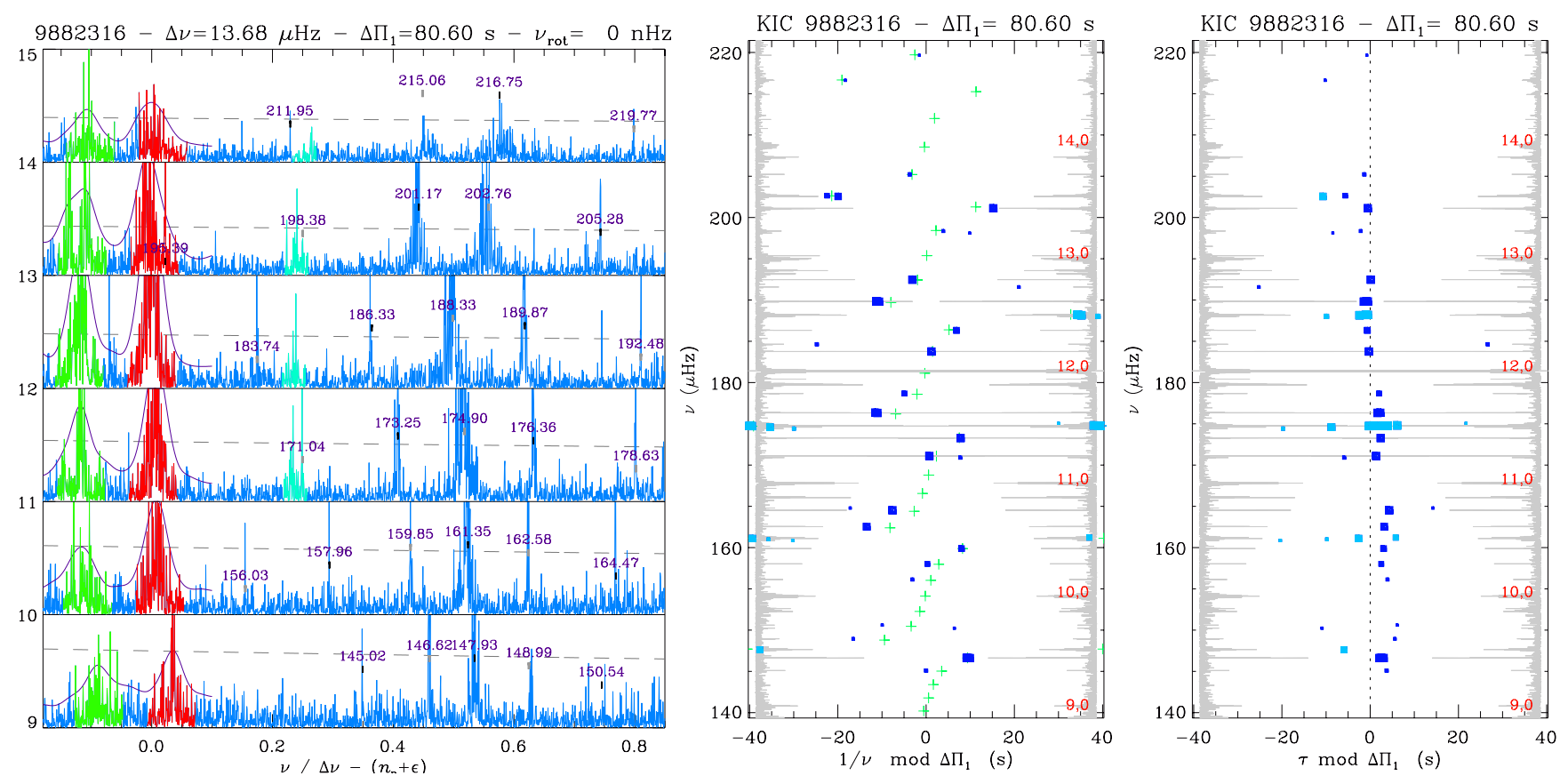

Fig. 5. Échelle diagrams of the RGB star KIC 9882316, which shows no rotational signature. Left: classical frequency échelle diagram as a function of the dimensionless frequency $v / \Delta v-\left(n_{\mathrm{p}}+\varepsilon\right)$. The radial order $n_{\mathrm{p}}$ is indicated on the $y$-axis. Radial modes (in red) are centred on 0 ; quadrupole modes (in green) near -0.12 , with a radial order $n_{\mathrm{p}}-1$; and $\ell=3$ modes (sometimes observed; in light blue) near 0.22 . Dipole mixed modes are identified with the frequency given by the asymptotic relation of mixed modes, in $\mu \mathrm{Hz}$. The fit is based on peaks showing a height greater than eight times the mean background value (grey dashed lines). Middle: classical period échelle diagram, where the abscissa is the period modulo the period spacing $\Delta \Pi_{1}$. The most prominent mixed modes, marked with blue filled squares (in light blue for peaks in the vicinity of $p-m$ modes), are automatically identified. In the background of the figure, the spectra are plotted twice and top to tail to make the mode identification easier, with the pressure radial orders indicated on the radial modes. Right: stretched period échelle diagram, where the abscissa is the stretched period $\tau$ modulo the period spacing $\Delta \Pi_{1}$. Pressure-dominated mixed modes are coded in light blue.

Table 1. Rotational splittings.

\begin{tabular}{rcccccccccc}
\hline \hline & \multicolumn{3}{c}{ Pressure modes } & \multicolumn{3}{c}{ Hand-made analysis } & \multicolumn{4}{c}{ Analysis based on stretched periods } \\
KIC & $v_{\max }$ & $\Delta v$ & $\Delta \Pi_{1}$ & $\mathcal{N}\left(v_{\max }\right)$ & $\delta v_{\text {rot,core }}{ }^{a}$ & $\Delta \tau_{m=1}$ & $\Delta \tau_{m=-1}$ & $\left\langle\Delta \tau_{m}\right\rangle$ & $x_{\text {rot }}$ & $\delta v_{\text {rot,core }}{ }^{b}$ \\
& $\mu \mathrm{Hz}$ & $\mu \mathrm{Hz}$ & $\mathrm{s}$ & & $\mathrm{nHz}$ & $\mathrm{s}$ & $\mathrm{s}$ & $\mathrm{s}$ & $\%$ & $\mathrm{nHz}$ \\
\hline 6144777 & 128.4 & 11.029 & 79.0 & 8.5 & 242 & 78.75 & 79.25 & 79.0 & 0.34 & 227 \\
9267654 & 118.0 & 10.358 & 78.0 & 9.5 & 490 & 77.30 & 78.55 & 77.9 & 0.75 & 523 \\
12008916 & 160.8 & 12.906 & 79.8 & 6.3 & 430 & 79.45 & 80.15 & 79.8 & 0.46 & 409 \\
\hline
\end{tabular}

Notes. ${ }^{(a)}$ Rotational splitting derived from Goupil et al. (2013). ${ }^{(b)}$ Rotational splitting derived from this work.

(Goupil et al. 2013). Conclusions remain the same, but estimating the separated contribution of the core and of the envelope then requires a separated study of $p-m$ and $g-m$ modes.

\subsection{Toward an automated determination of the global seismic parameters}

The method presented above has shown its ability to exhibit in a clear way the rotational structure of the mixed modes and to identify their azimuthal orders. This comes from the fact that Eq. (20) allows us to directly analyze the period pattern, and helps us to avoid the identification of the mixed modes prior to the measurement of $\Delta \Pi_{1}$ as in other methods (e.g., Datta et al. 2015). In many cases, and especially on the RGB, defining period spacings of dipole mixed modes requires first the full identification of the dipole modes, which is a severe drawback for complex spectra: rotational splittings, $\ell=3$ modes, and shortlived $p-m$ mode conspire against the identification of the angular degree and azimuthal order of the modes, then against the measurement of relevant $\Delta P$. The use of Eq. (20) alleviates this problem.

All stages used to draw the échelle diagrams were based on automated methods. The steps for obtaining $\Delta v$ were noted in Sect. 3 and are not modified by rotation. The asymptotic period spacings can also be obtained in an automated way (Vrard et al. $2015 b$ ). The next step is to profit from the views developed in this work to automate the measurement of the rotational splitting; this is the aim of a forthcoming paper. The method can also be used to investigate possible rapid rotators (Ouazzani et al. 2013). Possible complications arising from structural glitches are presented in the next section.

\section{Glitches}

In this section, we analyze how structural glitches modify the mixed-mode pattern and might complicate the oscillation spectrum. Glitches are due to rapid variation either in the sound-speed profile or in the Brunt-Väisälä profile. 
B. Mosser et al.: Period spacings in red giants. I.
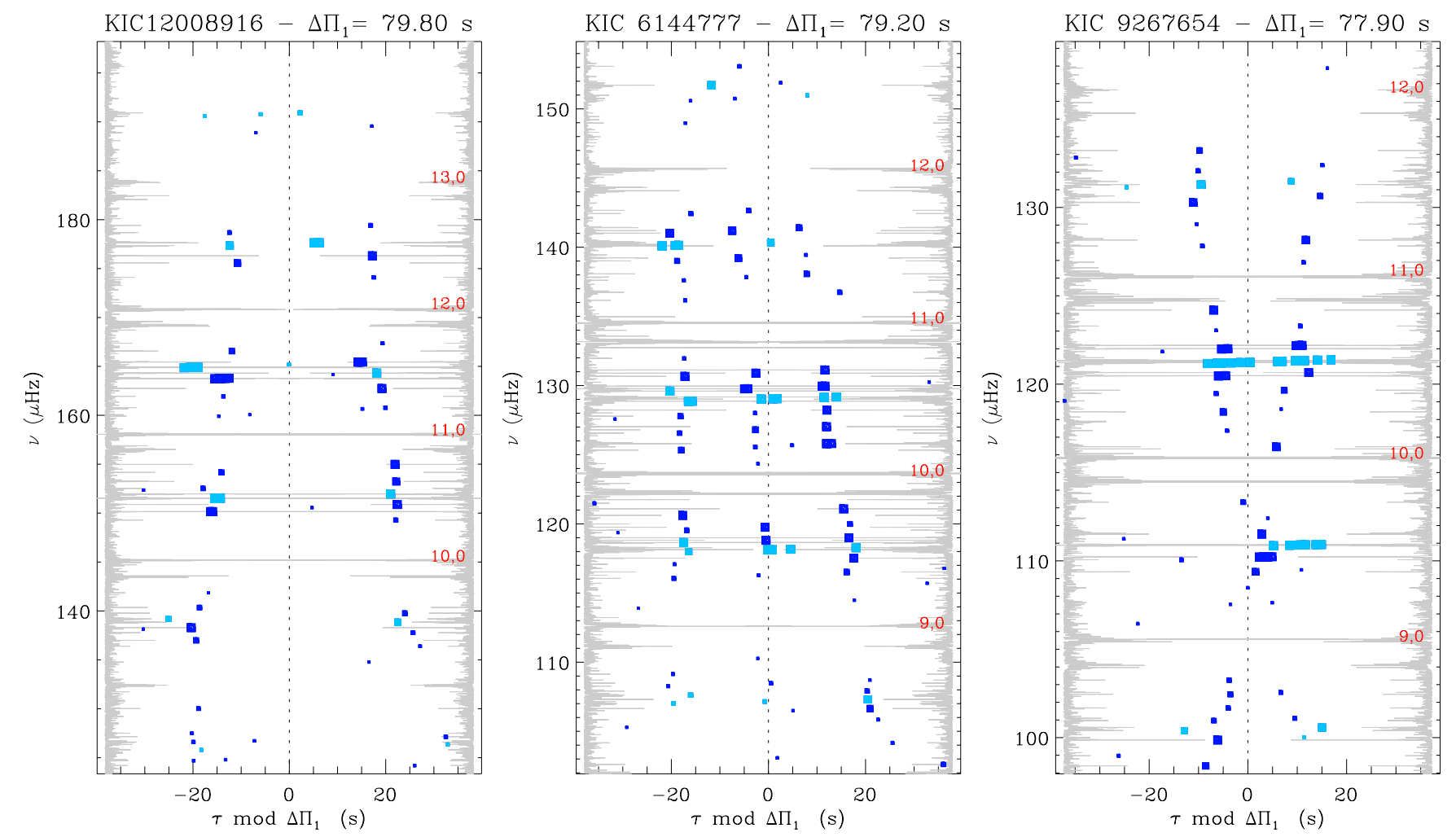

Fig. 6. Échelle diagrams based on the stretched periods of dipole mixed modes. The abscissae are the periods $\tau$ derived from Eq. (20), modulo the asymptotic period spacing $\Delta \Pi_{1}$; the ordinates are frequencies. The most prominent mixed modes, marked with filled squares, are automatically identified. In the background of the figure, the spectra are plotted twice and top to tail to make the mode identification easier, with the pressure radial orders indicated on the radial modes. From left to right we show RGB stars with different rotational splittings. Left: star seen equator-on where only $m= \pm 1$ modes are visible and with a moderate mean core rotation; they draw two ridges from which the rotation frequency $\delta v_{\text {rot }}$ can be derived. Middle: star seen with an inclination such that all three components of the rotational multiplets are visible and with a moderate mean core rotation. Right: star seen equator-on; as a consequence of the large mean core rotation, the ridges $m= \pm 1$ intersect.

\subsection{Sound-speed glitches}

The pressure mode spectrum of red giants is modulated by acoustic glitches due to abrupt variation of the first adiabatic exponent at the location of the second ionization of helium (Miglio et al. 2010; Broomhall et al. 2014). We have modeled the pressure glitches following Vrard et al. (2015a) with a simple sinusoidal modulation added to the pure pressure modes $v_{\mathrm{p}}$. We considered two cases corresponding to typical clump and RGB stars, and used the mean values of the glitch periods and amplitudes given in Vrard et al. (2015a): the periods of the glitch signature are in the range [3-4], in units of $\Delta v$, and the amplitude about a few percentage points, also in units of $\Delta v$. We note that the introduction of the sound-speed glitch does not modify the scheme presented in Sect. 2, so that one can easily retrieve the period spacings (Fig. 7).

The analysis of the stretched periods confirms that only $p-m$ modes are significantly affected by the sound-speed glitches. Their location and hence the value $d_{01}$ of the small separation in Eq. (7) must be precisely determined in order to perform a correct analysis of the glitch signature.

The influence on the $g-m$ modes is reduced since the pure gravity-mode pattern is not affected by the sound-speed glitch. According to the amplitude observed for acoustic glitches, the deviation in the $p-m$ mode is limited to a small fraction of $\Delta \Pi_{1}$. The relative amplitude $x_{\mathrm{p}}$ of the acoustic glitch, which corresponds to a frequency shift of $x_{\mathrm{p}} \Delta v$, translates into a period shift of the pure pressure modes about $x_{\mathrm{p}} \mathcal{N} \Delta \Pi_{1}$. The maximum period shift $\delta \tau_{\mathrm{p}}$ of the mixed modes is much smaller since the gravity component of the wave is not changed. We provide an estimate of this effect with a set of simulations of synthetic glitches performed at various evolutionary stages. Scaling relations of $\delta \tau_{\mathrm{p}}$ with $\Delta v, v_{\max }$, and $\Delta \Pi_{1}$ can be summarized by

$\delta \tau_{\mathrm{p}} \simeq 0.4 x_{\mathrm{p}} \mathcal{N}\left(v_{\max }\right)^{1 / 3} \Delta \Pi_{1}$.

Observed values of $x_{\mathrm{p}} / \Delta v$ are about 1-2\% (Vrard et al. 2015a), and at $v_{\max }$ there are about 10 gravity modes per $\Delta v$ frequency range so that the perturbation $\delta \tau_{\mathrm{p}} / \Delta \Pi_{1}$ is at most about $1-2 \%$. In all cases, sound-speed glitches are not expected to perturb the determination of the mixed-mode pattern or the measurement of $\Delta \Pi_{1}$.

\subsection{Buoyancy glitches}

For buoyancy glitches, we use the analysis of Miglio et al. (2008) describing them in slowly pulsating B stars and $\gamma$ Doradus stars, since the analytical expressions they derive are general enough to be applied to the red giant case. The asymptotic period spacing, modulated by a buoyancy glitch, can be written

$\Delta \Pi_{1 \text { glitch }}=\Delta \Pi_{1}(1+g(1 / v))$,

where $g$ is a periodic function. Its period is defined by

$\mathcal{G}=\frac{\Delta \Pi_{\mathrm{g}}}{\Delta \Pi_{1}}=\frac{\int_{r_{N 1}}^{r_{N 2}} N_{\mathrm{BV}} \frac{\mathrm{d} r}{r}}{\int_{r_{\mathrm{g}}}^{r_{N 2}} N_{\mathrm{BV}} \frac{\mathrm{d} r}{r}}$, 

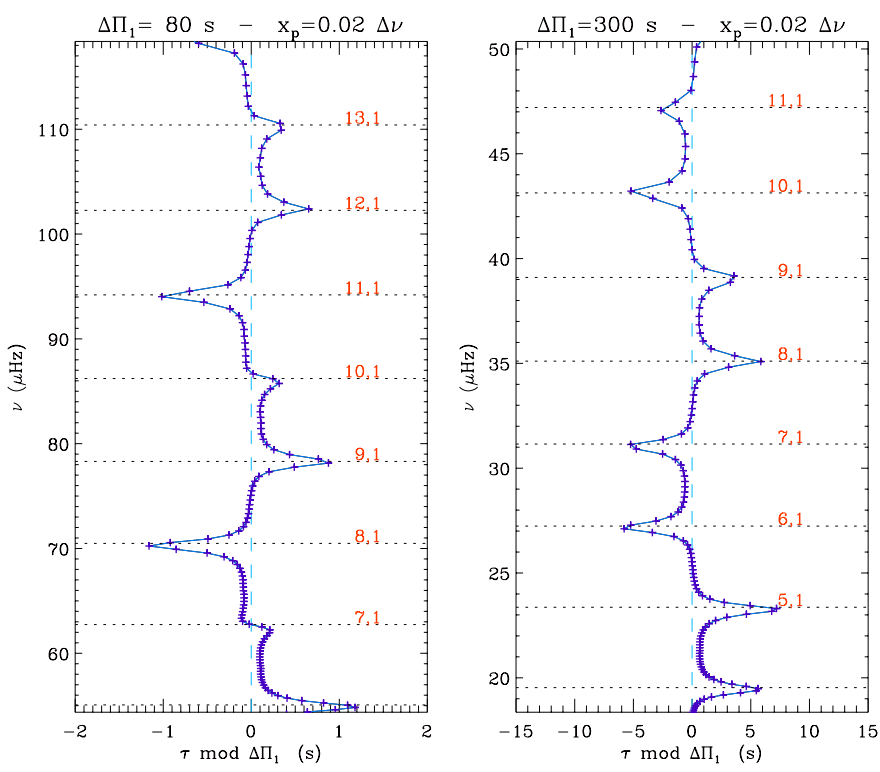

Fig. 7. Stretched period échelle diagrams of synthetic mixed-mode spectra including sound-speed glitches. Left: RBG star; Right: red clump star. Horizontal dotted lines indicate the location of the pure pressure dipole modes.

where $N_{\mathrm{BV}}$ is the Brunt-Väisälä frequency, $r_{N 1}\left(r_{N 2}\right)$ is the inner (outer) boundary of the radiative core, and where $r_{\mathrm{g}}$ is the radius of the buoyancy glitch ${ }^{1}$.

When Brunt-Väisälä glitches are present, the derivation of Eq. (1) must take the variation of $g$ into account in order to obtain Eq. (14). We suppose that the buoyancy glitch can be treated as a perturbation so that the influence of the glitch in the phase $\theta_{\mathrm{p}}$ can be neglected. Hence, Eq. (12) is changed into

$\frac{1}{\Delta \Pi_{1}} \frac{\mathrm{d} P}{\mathrm{~d} n_{\mathrm{m}}}=\zeta-\frac{\mathrm{d} g}{\mathrm{~d} n_{\mathrm{m}}}=\zeta_{\mathrm{g}}$.

The correcting term on the right-hand side of this equation includes the glitch contribution. We have checked that, except for huge glitches, the glitch-perturbed correction $\zeta_{\mathrm{g}}$ is very close to the glitch-free function $\zeta$ (Fig. 8). As a consequence, the function $\zeta$ can be safely used in Eq. (20) instead of $\zeta_{\mathrm{g}}$ to derive stretched periods. The stretched periods calculated with $\zeta$ exhibit the glitch signature; conversely, when $\zeta_{\mathrm{g}}$ is used in Eq. (20), the glitch signature is corrected. We also note that the expression used for the glitch is coherent with the modeling done by Cunha et al. (2015).

\subsection{Deep and shallow buoyancy glitches}

We analyzed the cases where the function $g$ expressing the buoyancy glitch varies sinusoidally and tested glitch periods $\mathcal{G}$ in a broad range from 1.01 to 100 . Even if the sine form is a simplification, it helps to investigate the glitch signature since it carries the most important information, namely the location of the glitch, which is related to the relative period $\mathcal{G}$. We denote the amplitude of the glitch as in Miglio et al. (2008): the amplitude $A$ corresponds to a relative jump $(1-A)$ in the Brunt-Väisälä profile at the glitch location.

\footnotetext{
1 This definition implies that the ratio $\mathcal{G}$ is necessarily larger than unity. It is also possible to change the boundaries of the denominator into, respectively, $r_{N 1}$ and $r_{\mathrm{g}}$ for another definition $\mathcal{G}^{\star}$ of the period, which verifies $1 / \mathcal{G}^{\star}=1-1 / \mathcal{G}$.
}

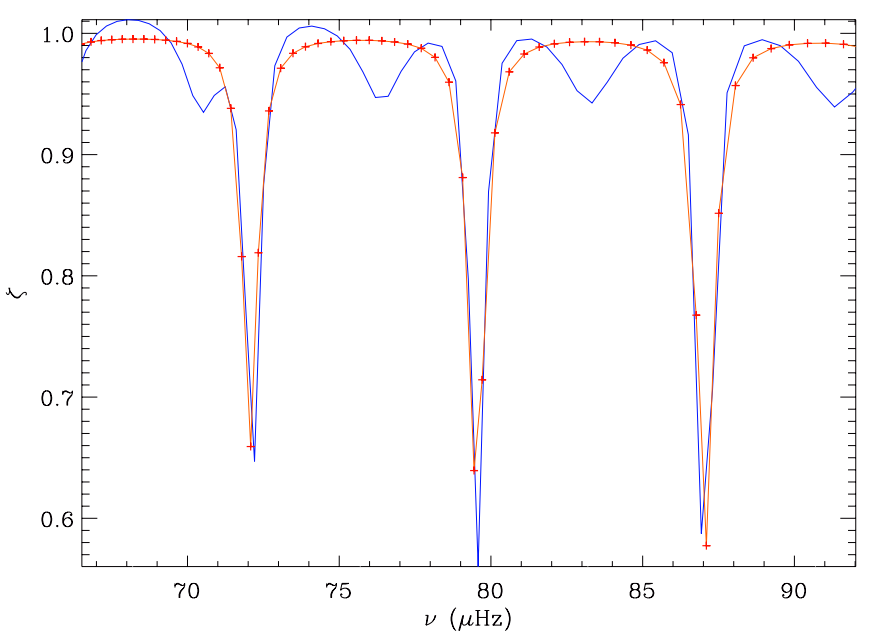

Fig. 8. Relative period spacing $\Delta P / \Delta \Pi_{1}$ (blue line) derived from the function $\zeta$ calculated without glitches (red line and plus signs). The values of $\zeta$ and $\zeta-\mathrm{d} g / \mathrm{d} n_{\mathrm{m}}$ are superimposed and cannot be distinguished.

Figure 9 shows that the échelle diagrams based on the stretched period spacings make it possible to retrieve the glitch profile. We note that the glitches with relative periods $(G-1)$ and $1 /(G-1)$ show similar modulation, except near the $p-m$ modes. Such a degeneracy in the glitch information is well known: for symmetry reasons, deep and shallow glitches cannot be distinguished. Here, the degeneracy is broken by the $p$ - $m$ modes. We are able to distinguish three main cases:

- For $\mathcal{G}$ values close to unity, that is for glitches located near the lower boundary of the Brunt-Väisälä cavity, the period bumping near the $p-m$ modes induces a significant shift compared to the period $\mathcal{G} \Delta \Pi_{1}$ (Eq. (26)). As a result, the large variation of the phase of the modulation exceeds $2 \pi$ which means that it cannot be corrected. So, a rapid variation of the stretched period near the $p-m$ modes is the signature of a shallow buoyancy glitch. The amplitude of the spikes at the $p-m$ modes can be used to investigate the amplitude of the modulation of the glitch. According to Fig. 9, the spikes near the $p-m$ modes have the same amplitude as the global modulation. This result is robust; it derives from a phase effect and is independent of the exact form of the periodic function $g$.

- For periods $G$ larger than 2 , the shift at the $p-m$ mode remains limited, so that the $\zeta$ contribution makes it possible to retrieve the glitch function $g(1 / v)$ in the échelle diagram based on the stretched period. Large values of $\mathcal{G}$ corresponding to shallow glitches show long-period modulations. The absence of any accident near the $p$ - $m$ mode in the échelle diagram is the signature of a shallow glitch.

- The case where $\mathcal{G}=2$ is an intermediate case: the glitch signature looks like a sawtooth profile; the varying amplitude of the modulation is a moiré effect. Determining the mean value of $\Delta \Pi_{1}$ may then be difficult. However, such cases were not encountered in the large data set analyzed in Mosser et al. (2014).

\subsection{An example of buoyancy glitch}

We consider the case of the red giant star KIC 9332840 observed by Kepler. This star has reached the red clump and shows a complex mixed-mode pattern (Mosser et al. 2012a). Two values of the period spacing were reported, and depend on the 

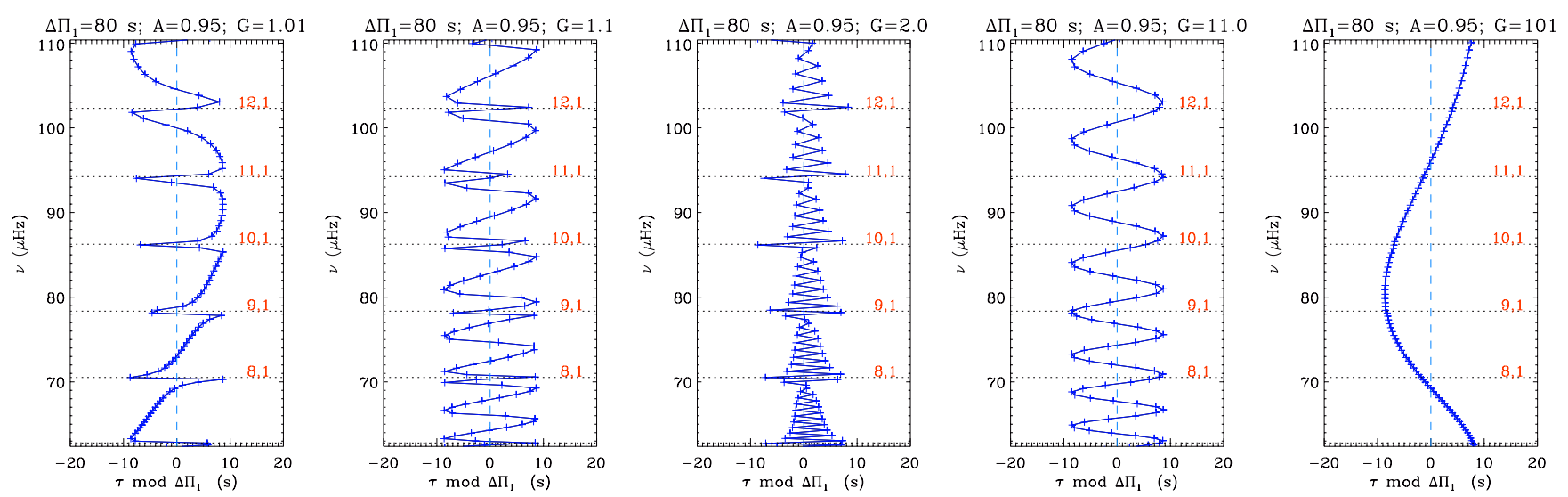

Fig. 9. Stretched period échelle diagrams of synthetic mixed-mode spectra including buoyancy glitches. The period of the buoyancy-glitch modulation is indicated for each plot; it increases from left to right. All other parameters are the same in all plots. Horizontal dotted lines indicate the location of the pure pressure dipole modes.

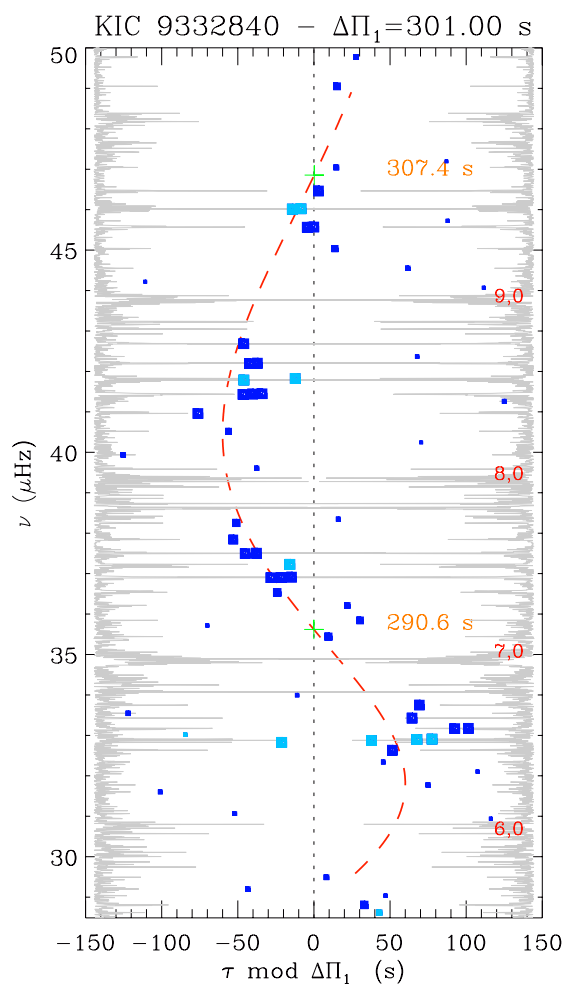

Fig. 10. Stretched period échelle diagram of KIC 9332840 showing a large-period modulation (dashed red line) compatible with a shallow buoyancy glitch. Peaks are identified as in Figs. 5 and 6. The minimum and maximum values of the varying period are indicated in orange.

frequency range: $298 \mathrm{~s}$ in the low-frequency range and $306 \mathrm{~s}$ in the high-frequency range. This variation can be attributed to the modulation induced by a buoyancy glitch (Fig. 10). An alternative explanation should be a deviation to the first-order asymptotic expansion. Observationally, the observed mixed modes have such high gravity radial orders, about 80 , that we do not favor this hypothesis. Theoretical simulations by Cunha et al. (2015) seem to confirm this for RGB stars.

When the modulation is interpreted as a buoyancy glitch, the stretched period diagram of this spectrum shows a signature with a high $G$ value in the range [40-50]. This means that the glitch has a shallow location and might be related to some accident in the Brunt-Väisälä profile near the outer boundary of the dense radiative core. The amplitude $A$, about $0.92 \pm 0.02$, provides a measure of the glitch contrast in the Brunt-Väisälä profile.

Unlike sound-speed glitches, buoyancy glitches may significantly perturb the mixed-mode pattern. However, according to previous observations (Mosser et al. 2014), this only occurs in a small minority of cases. A comprehensive observational study of buoyancy glitches also requires a systematic analysis of the red giant oscillation spectrum.

\section{Conclusion}

We rewrote the asymptotic expansion of dipole mixed modes in order to account for the most precise description of the pure pressure and pure gravity contributions. We showed that the bumped period spacing and frequency rotational splitting follow the same pattern constructed by the function $\zeta$ (Deheuvels et al. 2015). This equality implies that the information present in the rotation splittings and in the period spacings is degenerated: both signatures are derived from the ratio of the kinetic energy in the radiative core compared to the total kinetic energy of the modes. Then, we showed how the function $\zeta$ can be used to stretch the mixed-mode pattern and to derive the contribution of the pure gravity-mode pattern. In fact, stretched periods mimic the pure gravity period spacing: they are evenly spaced, and the mean spacing corresponds to the asymptotic period spacing $\Delta \Pi_{1}$. As a result, each component of the rotational multiplets can be identified and the asymptotic period spacing can be measured even in the presence of important rotational splittings.

It follows that all steps of the red giant spectrum analysis can be automated, since the complex mixed-mode forest is now as regular as an artificially planted thicket. We have derived an automated method for measuring $\Delta \Pi_{1}$ in a companion paper (Vrard et al. 2015b); the automated measurement of the core rotation in 13000 red giant spectra observed by Kepler and showing solar-like oscillations is in progress.

Another output is the possible interpretation of the modulation of the period spacing as buoyancy glitches. Échelle diagrams based on the stretched periods are able to put them in evidence. This reinforces the capability of mixed modes for probing the stellar cores and inferring unique information on the physical conditions in the nuclear-burning region.

Acknowledgements. We acknowledge the entire Kepler team, whose efforts made these results possible. We acknowledge financial support from the 
Programme National de Physique Stellaire (CNRS/INSU) and from the ANR program IDEE Interaction Des Étoiles et des Exoplanètes. We thank the referee, H. Shibahashi, for his constructive comments.

\section{Appendix A: Limiting cases}

\section{A.1. Gravity-dominated mixed modes}

The frequency $v$ of a gravity-dominated mixed mode ( $g$ - $m$ mode) is significantly offset compared to the pure pressure frequency $v_{\mathrm{p}}$, so that its phase $\theta_{\mathrm{p}}$ is close to $\pi / 2$, modulo $\pi$, and its cosine is small. Hence, the function $\zeta$ shows a wide variation. For these $g-m$ modes, $\tan \theta_{\mathrm{p}}$ is large so that $\tan \theta_{\mathrm{g}}$ is necessarily large too, according to the asymptotic relation (Eq. (1)). Considering that both cosine values are small, they can be approximated by the inverse of their tangents. As a result, when taking the asymptotic expansion into account, the value of $\zeta$ for $g-m$ modes is close to $\zeta_{\max }$ defined by

$\zeta_{\max }=\left[1+q \frac{v^{2} \Delta \Pi_{1}}{\Delta v\left(n_{\mathrm{p}}\right)}\right]^{-1}=\left[1+\frac{q}{\mathcal{N}}\right]^{-1}$,

hence close to unity in most cases except in the lower part of the red giant branch. As a by-product of this discussion, we note that the $g-m$ periods are close to $\left(n_{\mathrm{m}}+1 / 2+\varepsilon_{\mathrm{g}}\right) \Delta \Pi_{1}$. So, even if $g-m$ modes behave like gravity modes, their frequencies are shifted due to an extra phase added by the coupling; this situation resembles the $\pi$ phase added to the wavefront of an optical ray crossing a focal point.

\section{A.2. Pressure-dominated mixed modes}

On the contrary, for pressure-dominated $(p-m)$ mixed modes, the phase $\theta_{\mathrm{p}}$ is close to 0 : the function $\zeta$ shows reduced variation between $\zeta_{\min }$ and 1 . The low value of $\tan \theta_{\mathrm{p}}$ implies the low value of $\tan \theta_{\mathrm{g}} ; \cos ^{2} \theta_{\mathrm{p}}$ and $\cos ^{2} \theta_{\mathrm{g}}$ are both close to unity, so that for $p-m$ modes $\zeta$ is found to be close to $\zeta_{\min }$ defined by

$\zeta_{\min }=\left[1+\frac{1}{q} \frac{v^{2} \Delta \Pi_{1}}{\Delta v\left(n_{\mathrm{p}}\right)}\right]^{-1}=\left[1+\frac{1}{q \mathcal{N}}\right]^{-1}$.

This term $\zeta_{\min }$ is significantly different from unity; as is well known, period spacings of $p-m$ modes cannot be used to derive the asymptotic period spacing (e.g., Bedding et al. 2011; Stello et al. 2013).

\section{References}

Beck, P. G., Montalban, J., Kallinger, T., et al. 2012, Nature, 481, 55 Bedding, T. R., Huber, D., Stello, D., et al. 2010, ApJ, 713, L176 Bedding, T. R., Mosser, B., Huber, D., et al. 2011, Nature, 471, 608 Belkacem, K., Marques, J. P., Goupil, M. J., et al. 2015a, A\&A, 579, A31 Belkacem, K., Marques, J. P., Goupil, M. J., et al. 2015b, A\&A, 579, A30 Benomar, O., Belkacem, K., Bedding, T. R., et al. 2014, ApJ, 781, L29 Broomhall, A.-M., Miglio, A., Montalbán, J., et al. 2014, MNRAS, 440, 1828

Chaplin, W. J., Kjeldsen, H., Christensen-Dalsgaard, J., et al. 2011, Science, 332, 213

Christensen-Dalsgaard, J. 2012, in Progress in Solar/Stellar Physics with Helioand Asteroseismology, eds. H. Shibahashi, M. Takata, \& A. E. Lynas-Gray, ASP Conf. Ser., 462, 503

Christensen-Dalsgaard, J., Silva Aguirre, V., Elsworth, Y., \& Hekker, S. 2014, MNRAS, 445, 3685

Corsaro, E., De Ridder, J., \& García, R. A. 2015, A\&A, 579, A83

Cunha, M. S., Stello, D., Avelino, P. P., Christensen-Dalsgaard, J., \& Townsend, R. H. D. 2015, ApJ, 805, 127

Datta, A., Mazumdar, A., Gupta, U., \& Hekker, S. 2015, MNRAS, 447, 1935

De Ridder, J., Barban, C., Baudin, F., et al. 2009, Nature, 459, 398

Deheuvels, S., García, R. A., Chaplin, W. J., et al. 2012, ApJ, 756, 19

Deheuvels, S., Doğan, G., Goupil, M. J., et al. 2014, A\&A, 564, A27

Deheuvels, S., Ballot, J., Beck, P. G., et al. 2015, A\&A, 580, A96

Goupil, M. J., Mosser, B., Marques, J. P., et al. 2013, A\&A, 549, A75

Kallinger, T., Mosser, B., Hekker, S., et al. 2010, A\&A, 522, A1

Kallinger, T., Hekker, S., Mosser, B., et al. 2012, A\&A, 541, A51

Marques, J. P., Goupil, M. J., Lebreton, Y., et al. 2013, A\&A, 549, A74

Michel, E., Baglin, A., Auvergne, M., et al. 2008, Science, 322, 558

Miglio, A., Montalbán, J., Noels, A., \& Eggenberger, P. 2008, MNRAS, 386, 1487

Miglio, A., Montalbán, J., Carrier, F., et al. 2010, A\&A, 520, L6

Montalbán, J., Miglio, A., Noels, A., et al. 2013, ApJ, 766, 118

Mosser, B., \& Appourchaux, T. 2009, A\&A, 508, 877

Mosser, B., Belkacem, K., Goupil, M., et al. 2010, A\&A, 517, A22

Mosser, B., Barban, C., Montalbán, J., et al. 2011a, A\&A, 532, A86

Mosser, B., Belkacem, K., Goupil, M., et al. 2011b, A\&A, 525, L9

Mosser, B., Elsworth, Y., Hekker, S., et al. 2012a, A\&A, 537, A30

Mosser, B., Goupil, M. J., Belkacem, K., et al. 2012b, A\&A, 540, A143

Mosser, B., Goupil, M. J., Belkacem, K., et al. 2012c, A\&A, 548, A10

Mosser, B., Michel, E., Belkacem, K., et al. 2013, A\&A, 550, A126

Mosser, B., Benomar, O., Belkacem, K., et al. 2014, A\&A, 572, L5

Ouazzani, R.-M., Goupil, M. J., Dupret, M.-A., \& Marques, J. P. 2013, A\&A, 554, A 80

Provost, J., \& Berthomieu, G. 1986, A\&A, 165, 218

Shibahashi, H. 1979, PASJ, 31, 87

Stello, D., Huber, D., Bedding, T. R., et al. 2013, ApJ, 765, L41

Tassoul, M. 1980, ApJS, 43, 469

Unno, W., Osaki, Y., Ando, H., Saio, H., \& Shibahashi, H. 1989, Nonradial oscillations of stars (Tokyo: Univ. of Tokyo Press)

Vrard, M., Mosser, B., Barban, C., et al. 2015a, A\&A, 579, A84

Vrard, M., Mosser, B., Samadi, R., et al. 2015b, A\&A, submitted 